\title{
Space-Time-Waveform Adaptive Processing for Frequency Diverse Distributed Radar Apertures
}

\author{
Raviraj S. Adve, Lorne Applebaum \\ Dept. of Elec. and Comp. Eng., \\ University of Toronto \\ 10 King's College Road, Toronto, \\ Ontario, M5S 3G4, Canada \\ rsadve@comm. utoronto.ca, \\ lorne.applebaumeutoronto.ca
}

\author{
Michael C. Wicks \\ US Air Force Research Laboratory, \\ Sensors Directorate \\ 26 Electronics Parkway, \\ Rome, NY 13441, USA. \\ wicksmerl.af.mil
}

\author{
Richard A. Schneible \\ Stiefvater Consultants \\ 10002 Hillside Terrace, \\ Marcy, NY 13403, USA. \\ Richard.Schneibleerl.af.mil
}

\begin{abstract}
This paper reviews recent developments in the field of adaptive processing for frequency diverse, distributed, radar apertures. The large baseline of such a distributed radar results in angular resolution that is orders of magnitude better than the resolution of a single large radar. This capability comes at the cost of grating lobes (multistatics with evenly spaced apertures) or high sidelobes (multistatics with randomly spaced apertures). This paper presents some of the issues specific to such a radar and develops the notion of frequency diversity and presents the outlines of a data model that addresses the specific nature of frequency diverse distributed apertures.
\end{abstract}

\section{INTRODUCTION}

Surveillance radar systems operate in a severe and dynamic interference environment. The interference is a sum of clutter, possible deliberate electronic counter measures (ECM) and noise. Space-time adaptive processing (STAP) has been well accepted as the best means to detect weak targets in such interference [1]. In STAP, signals received over space and time are jointly processed to suppress interference and enhance target signatures at an assumed look angle and Doppler frequency. More recently, researchers have begun exploring extending the notion of diversity to radar systems, using a distributed radar [2]. The phrase "waveform diversity" has now come to include distributed communication networks, distributed space-time coding, and distributed target detection. Our focus here is target detection using a distributed radar.

A distributed radar comprises a network of transmit/receive apertures placed relatively distant from one other. Unlike traditional radar systems, these apertures could be many thousands of wavelengths apart. The distributed radar operates in a multistatic mode with several of the apertures transmitting (the same signal, different uncorrelated signals or orthogonal signals) simultaneously. Similarly, several of the apertures receive and process the returned signals due to all transmissions. Some of the transmissions may be due to illuminators of opportunity, such as television stations or cellular communication traffic [3]. The extremely large baseline in such a multistatic radar results in extremely narrow antenna beams, in turn providing angular resolution orders of magnitude better than that of a monolithic system (single large radar) with the same power-aperture product. This improved target tracking and interference rejection comes at the cost of grating lobes (multistatics with evenly spaced apertures) or high sidelobes (multistatics with randomly spaced apertures).

This paper discusses a waveform diverse multistatic radar. Consider a distributed radar system comprising $N$-apertures. Waveform diversity is implemented as a set of orthogonal, relatively narrowband signals, offset in center frequency. Each element receives and processes the returns from all $N$ transmissions - each fast time sample (corresponding to each range cell) for each pulse therefore corresponds to a $N^{2}$ vector.

In such a system, there are a few unique concepts [4]:

- Adaptive space/waveform processing: Traditionally, adaptive processing has focused on the space and time dimensions. In a distributed aperture, the waveform dimension augments the traditional space-time-range data cube. This has implications for the adaptive process with the increased degrees of freedom (DOF) providing greater performance, but also requiring larger training sets. The use of frequency diverse transmissions alleviates this problem since the returns are orthogonal over all ranges. However this, as we will show later, requires coherent processing across frequencies.

- Steering Vectors: The spatial steering vector is related to the look direction while the temporal steering vector is determined by the look Doppler frequency. In our case, the time dimension is augmented with the waveform dimension. The space/waveform steering vector is determined by the look angle uniquely with a different spatial steering vector for each transmit frequency.

- True Time Delay: The use of multiple frequencies, and the fact that the steering vector is frequency dependent, precludes use of phase shifts for beam steering. Maximizing signal power at a potential target point requires use of true time delay processing to focus the overall radar aperture.

- Spacing of subapertures/frequencies: Distributing the apertures and separating the transmit frequencies introduces two new degrees of freedom available to the 
radar designer: the spacing between the antenna elements and the frequencies. Equally spaced elements with equally spaced frequencies can lead to grating lobes that can reduce the effectiveness of the adaptive process. More practically, frequency diversity allows for adaptive choice of the transmission frequencies to achieve a signal processing purpose, such as maximizing overall output signal-to-interference-plus-noise ratio (SINR).

- Targets/interference are not in the far field: By common definition, for an antenna of total length $D$, the far field region is determined by three conditions: $R>\lambda, R>D$ and $R>D^{2} / \lambda$ where $\mathrm{R}$ is the radial distance and $\lambda$ is the wavelength of operation [5]. From a physical point of view, the far field may be defined as the region where the spatial steering vector is effectively independent of the radial distance. In our numerical example below, we choose $D=200 \mathrm{~m}$ with a center frequency $10 \mathrm{GHz}$, setting the beginning of the far field at approximately $1500 \mathrm{~km}$. The target and interference are therefore not necessarily in the far field. This has serious implications in the type of adaptive processing scheme chosen, including choice of secondary data to estimate the interference covariance matrix. Similar to STAP for bistatic radar, this range dependent steering vector reduces the secondary data available to estimate the covariance matrix [6], [7].

This paper is organized as follows: Section II presents a recently developed model for the data received by a frequency diverse, distributed, aperture. Section III presents some results using the data model of Section II. Finally, Section IV describes some of the recent trends and future work in this area.

\section{Data Model for Frequency Diverse Apertures}

In the development of STAP, an important moment was the availability of an effective data model for the target, clutter, ECM and noise signals [1]. This section presents a model to generate data for the case of frequency diverse, distributed apertures. The development here is taken largely from [8].

Consider $N$ sub-apertures distributed on the $x-y$ plane at points $\left(x_{n}, y_{n}\right), n=1, \ldots, N$. Each aperture transmits on a carrier frequency $f_{n}$ modulated with $M$ linear-FM pulses within a coherent pulse interval (CPI). To focus on a look point $\left(X_{t}, Y_{t}, Z_{t}\right)$ a delay is added to each element's transmitted signal. The true time delay for the $n^{\text {th }}$ element is

$$
\Delta T_{n}=\frac{\max \left\{D_{n}\right\}-D_{n}}{c},
$$

where $D_{n}$ is the distance between the $n^{\text {th }}$ element and the look point, and $c$ is the speed of light. Independently delaying the received signals allows the time associated with the look point range gate to be identical for all elements.

The signal transmitted by the $n^{\text {th }}$ element is given by

$$
s(t)=u(t) e^{j 2 \pi f_{n} t+j \psi_{n}} ; u(t)=\sum_{m=0}^{M-1} u_{p}\left(t-m T_{r}\right),
$$

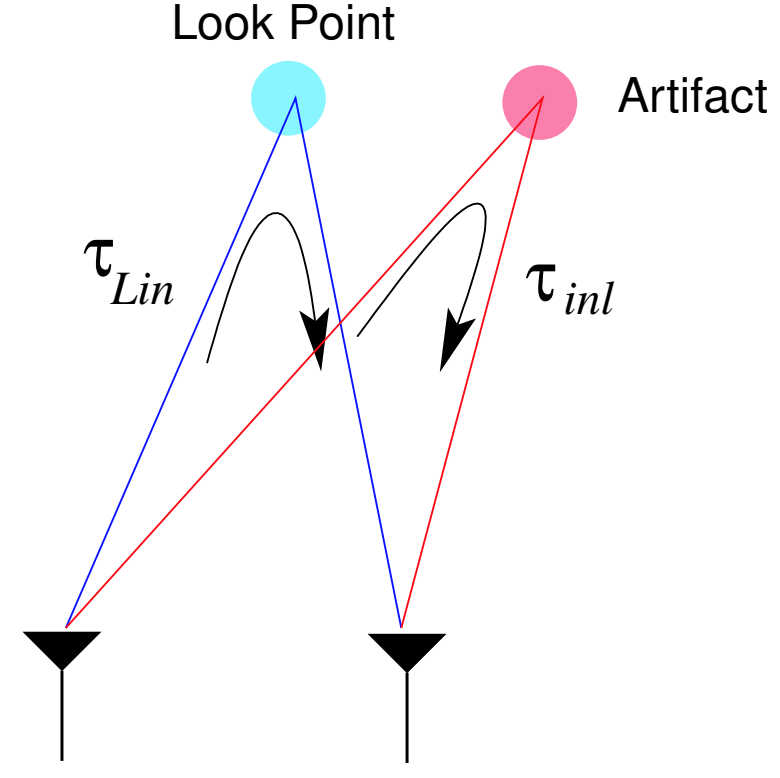

Fig. 1. Illustration of $\tau_{i n l}$ and $\tau_{L i n}$

where $u(t)$ is the complex envelope of $M$ linear-FM pulses with pulse repetition interval (PRI) $T_{r}$.

Consider a reflecting artifact (target or clutter) labelled by index $l$ at $\left(X_{l}, Y_{l}, Z_{l}\right)$, which is not necessarily the look point. The time taken for the signal to reach the artifact from the $n^{\text {th }}$ transmitting element is $\tau_{n l}^{\mathrm{Tx}}=$ $\left(\sqrt{\left(x_{n}-X_{l}\right)^{2}+\left(y_{n}-Y_{l}\right)^{2}+Z^{2}}\right) / c$. Similarly, the signal takes $\tau_{i l}^{\mathrm{Rx}}=\left(\sqrt{\left(x_{i}-X_{l}\right)^{2}+\left(y_{i}-Y_{l}\right)^{2}+Z_{l}^{2}}\right) / c$ to propagate from the artifact to the $i^{\text {th }}$ receiver. Thus, the received signal at element $i$ is

$$
r_{i n l}(t)=A_{n l} u\left(t-\tau_{n l}^{\mathrm{Tx}}-\tau_{i l}^{\mathrm{Rx}}\right) e^{j 2 \pi\left(f_{n}+f_{d n l}\right)\left(t-\tau_{n l}^{\mathrm{Tx}}-\tau_{i l}^{\mathrm{Rx}}\right)},
$$

where $A_{n l}$ is a complex amplitude (with $\psi_{n}$ absorbed into its random phase) and $f_{d n l}$ is the Doppler frequency induced by the motion of the artifact and is a function of carrier frequency. Note that, although the artifacts are not necessarily in the far field, they are assumed to be far enough that the Doppler frequency seen by each element is approximately the same.

After down conversion, matched filtering and receiver delay, the signal from a single artifact becomes

$$
\begin{aligned}
x_{i n l}(t)=A_{n l} e^{-j 2 \pi f_{n} \tau_{i n l}} \sum_{m=0}^{M-1} e^{j 2 \pi f_{d n l} m T_{r}} & \\
& \times \chi\left(t-m T_{r}-\tau_{i n l}-\Delta T_{i}, f_{d n l}\right),
\end{aligned}
$$

where $\tau_{i n l}=\tau_{n l}^{\mathrm{Tx}}+\tau_{i l}^{\mathrm{Rx}}$ and $\chi(t, f)$ is the ambiguity function of the linear-FM pulse.

Consider the processing of only the $m^{\text {th }}$ pulse. To focus on the look point, the receiver will range gate in such a way that the temporal term of the ambiguity function is zero for an artifact at the look point. That is, for all artifacts, $x_{i n l}(t)$ 
is sampled at the time

$$
t=m T_{r}+\tau_{L i n}+\Delta T_{i},
$$

where $\tau_{L i n}$ is the total travel time from the $n^{\text {th }}$ transmitter to the look point to the $i^{\text {th }}$ receiver of the signal. $\tau_{L i n}$ and $\tau_{i n l}$ are illustrated in Figure 1. The sample for the $m^{\text {th }}$ pulse is the sum of the contributions of all artifacts and is

$$
\begin{aligned}
x_{i n m}=\sum_{l} A_{n l} e^{-j 2 \pi f_{n} \tau_{i n l}} e^{j 2 \pi f_{d n l} m T_{r}} & \\
& \times \chi\left(\tau_{\text {Lin }}-\tau_{i n l} f_{d n l}\right) .
\end{aligned}
$$

Space-Time Adaptive Processing (STAP) uses the interference covariance matrix to obtain adaptive weights that detect a weak target in stronger interference [1]. The fundamental step in STAP is, therefore, obtaining the interference covariance matrix. In practice, the interference matrix is usually estimated using "secondary" data from range cells outside the range cell of interest (the primary range cell). The applicability of STAP to the above model is tested here by comparing the performance using the true interference covariance matrix with the performance using an estimated covariance matrix. The examples presented also illustrate the importance of coherent processing across the multiple frequencies.

\section{A. Properties of Signal Statistics}

Although down conversion eliminates the carrier signals, the signal samples from different carriers are statistically orthogonal. This is a result of the phase $\psi$ in Eqn. (1), as well as the phase of $A_{n l}$ induced by reflection in Eqn. (2). These phases are assumed to be random and uncorrelated across the frequencies.

Since the signals from each frequency are orthogonal, STAP can be performed on them independently. As a result, length$M N$ spatial-temporal sample snapshots can be used with STAP rather than the length- $N^{2} M$ vectors. These snapshots are column vectors denoted by $\mathrm{x}$ with elements described by Eqn. (4) for a given frequency. The elements of the vector span the samples from the $M$ pulses within a coherent pulse interval (CPI) for each of the $N$ receiving sub-apertures. In the following sections, the subscripts indicating transmitting element will be dropped since it is understood that each frequency is processed independently.

\section{B. Interference Covariance Matrix}

The true interference covariance matrix is given by $\mathbf{R}_{c}=$ $E\left\{\mathbf{x}_{c} \mathbf{x}_{c}^{H}\right\}$, where $\mathbf{x}_{c}$ corresponds to the snapshot of the signal composed only of interfering artifacts, ${ }^{H}$ denotes the conjugate transpose (Hermitian) and $E\{\cdot\}$ denotes the statistical expectation operator [1]. Note that the target itself is considered an interfering artifact at all look points other than its location. If there are $N_{c}$ clutter artifacts with independently random phases in $A_{l}$, then from Eqn. (4)

$$
\begin{aligned}
\left\{\mathbf{R}_{c}\right\}_{p q}=\sum_{l=1}^{N_{c}} & \left|A_{l}\right|^{2} e^{j 2 \pi f_{n}\left(\tau_{\alpha l}-\tau_{i l}\right)} e^{j 2 \pi f_{d l} T_{r}(m-\beta)} \\
& \times \chi\left(\tau_{L i}-\tau_{i l}, f_{d l}\right) \chi^{*}\left(\tau_{L \alpha}-\tau_{\alpha l}, f_{d l}\right),
\end{aligned}
$$

with the $p^{\text {th }}$ element of $\mathbf{x}_{c}$ corresponding to the element $i$ and pulse $m$ and the $q^{\text {th }}$ element corresponding to element $\alpha$ and pulse $\beta$. Note that $\mathbf{R}_{c}$ depends on the look point.

To estimate the covariance matrix, samples straddling the look point range gate are used. The signal in Eqn. (3) is sampled $K$ times at $t_{k}=m T_{r}+\tau_{L i n}+\Delta T_{i}+k T_{s}$, where the integer $k \in[-\lceil K / 2\rceil,\lfloor K / 2\rfloor]$ and $T_{s}$ is the sample period. The estimated interference covariance matrix $\hat{\mathbf{R}}_{c}$ is obtained in the usual manner [1]. If $\mathbf{x}_{k}$ represents the $k^{\text {th }}$ sample,

$$
\hat{\mathbf{R}}=\frac{1}{K} \sum_{k=1}^{K} \mathbf{x}_{k} \mathbf{x}_{k}^{H}
$$

\section{Steering Vector}

The steering vector $\mathbf{s}$ is defined by the look point and Doppler bank frequency. The element of $\mathbf{s}$ corresponding to sub-aperture $i$ and pulse $m$ has the value

$$
s_{i m}=e^{-j 2 \pi f_{n} \tau_{i l}} e^{j 2 \pi f_{d} m T_{r}} \chi\left(0, f_{d}\right) .
$$

\section{Modified Sample Matrix Inversion}

With either the true or estimated covariance matrix, modified sample matrix inversion (MSMI) [1] is used for target detection. Reintroducing subscripts indicating transmitted frequency, the formula used for MSMI statistics is

$$
\operatorname{MSMI}=\frac{\left|\sum_{n=1}^{N} \mathbf{w}_{n}^{H} \mathbf{x}_{n}\right|^{2}}{\left|\sum_{n=1}^{N} \mathbf{w}_{n}^{H} \mathbf{s}_{n}\right|},
$$

where $\mathbf{w}_{n}$ are the STAP weight vectors for each frequency. Equation (8) combines the processing of multiple frequencies and is derived by applying traditional MSMI on the $N^{2} M$ length vectors formed by stacking $\mathbf{x}_{n}, \mathbf{s}_{n}$ and $\mathbf{w}_{n}$.

\section{E. Doppler Banks}

In processing the received signal, we select a certain Doppler frequency. It is important to note, however, that the Doppler frequency for each carrier is different for a given target velocity. Thus, it is more appropriate to think of velocity banks rather than conventional Doppler banks. We combine the outputs from the corresponding velocity banks from each carrier to produce the system output. That is, the banks are organized by velocity rather than frequency.

\section{NUMERICAL RESULTS}

This section illustrates some of the main issues when dealing with frequency diverse, distributed apertures using the model developed above. The first example illustrates the use of element position to dampen grating lobes. Due to the extremely large baselines, grating lobes are a significant problem when using equally spaced distributed apertures. In [4] it was suggested a random placement of apertures would help alleviate this problem. However, the formulation there did not account for the fact that the targets and clutter sources are in the near field of the array. 
The second example uses the model developed in Section II include the impact of interference and presents the interference suppression capabilities of the two approaches.

The examples use the simulation scenario as in [8]. The system comprises a 16 element array distributed over a $200 \mathrm{~m} \times$ $200 \mathrm{~m}$ grid at the origin on the $x-y$ plane. Above the array, is a target and interfering clutter. The target was modelled as a point reflector with a signal-to-noise ratio (SNR) of $10 \mathrm{~dB}$. The clutter is modelled as a ball of random low power sources with a radius of $200 \mathrm{~m}$ and a centre separated from the target by $800 \mathrm{~m}$. Table I summarizes the simulation parameters.

TABLE I

SimUlation PARAMETERS

\begin{tabular}{|c||c|}
\hline \multicolumn{1}{|c||}{ Parameter } & Value \\
\hline \hline$N$ & 16 \\
\hline$M$ & 8 \\
\hline Up Chirp Bandwidth & $10 \mathrm{MHz}$ \\
\hline Up Chirp Duration & $10 \mu \mathrm{s}$ \\
\hline PRI & $50 \mu \mathrm{s}$ \\
\hline Inter-element Frequency Offset & $100 \mathrm{MHz}$ \\
\hline Target SNR & $10 \mathrm{~dB}$ \\
\hline Target Velocity & $50 \mathrm{~m} / \mathrm{s}$ \\
\hline Clutter INR & $50 \mathrm{~dB}$ \\
\hline Target Location & $(476.9 \mathrm{~m},-60.0 \mathrm{~m}, 200 \mathrm{~km})$ \\
\hline
\end{tabular}

\section{A. Example 1: Target Response}

This example illustrates the benefits of using a frequency diverse, distributed aperture with elements randomly spaced. The figures below present the target response as a function of look location, here along the $x$-axis. This is effectively the overall array beampattern for a frequency diverse, distributed aperture.

Figure 2 plots the target pattern an equispaced array (over the rectangular domain) with all elements transmitting at a single frequency. The grating lobes are clear, though note the slight taper in the grating lobe pattern due to the focusing on the single target look point. Figure 3 plots the target response for a frequency diverse equispaced aperture. The impact of frequency diversity is clear - the locations of the grating lobes for each of the 16 frequencies is slightly different, resulting in significant suppression of these undesired grating lobes. The use of true time delay focuses the energy from all 16 frequencies (elements) at the look point.

Figure 4 plots the target response for a frequency diverse aperture with elements spaced randomly over the $200 \mathrm{~m} \times$ $200 \mathrm{~m}$ total aperture. The dramatic improvement over the earlier figures, including Fig. 3, is clear. Randomization of the array element locations randomizes the locations of the sidelobes at each frequency (the notion of grating lobes is not well defined for a randomly placed array), in turn minimizing the target response sidelobes.

\section{B. Example 2: Horizontal Resolution}

In [8] the data model is used to illustrate the importance of phase coherence across multiple frequencies. Another characteristic of the system is that, due to the true time delay

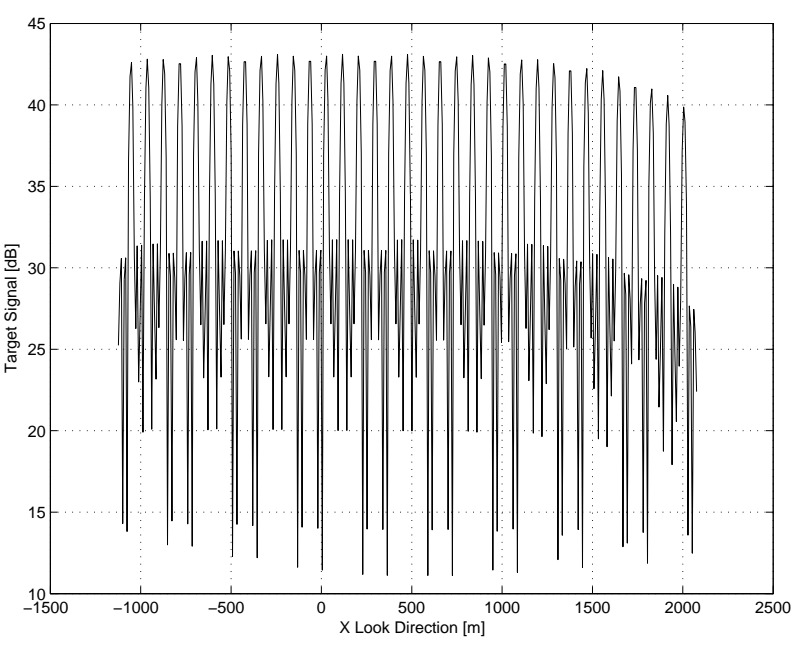

Fig. 2. Target return for an equispaced array at a single frequency

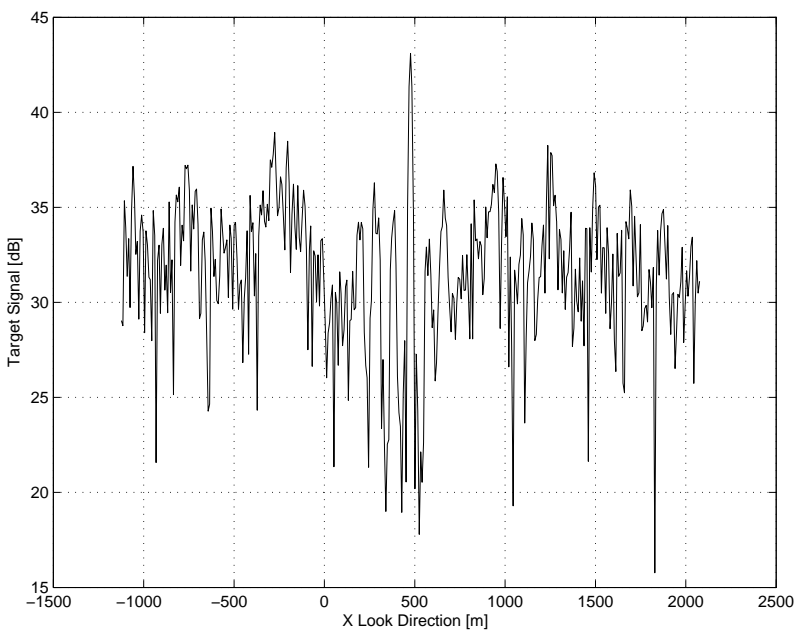

Fig. 3. Target return for an equispaced array including frequency diversity

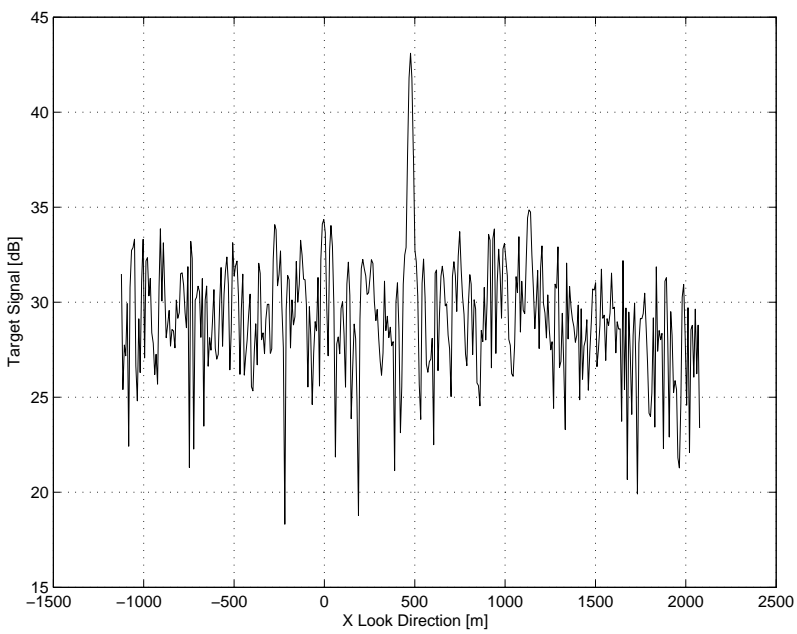

Fig. 4. Target return for randomly spaced apertures including frequency diversity 


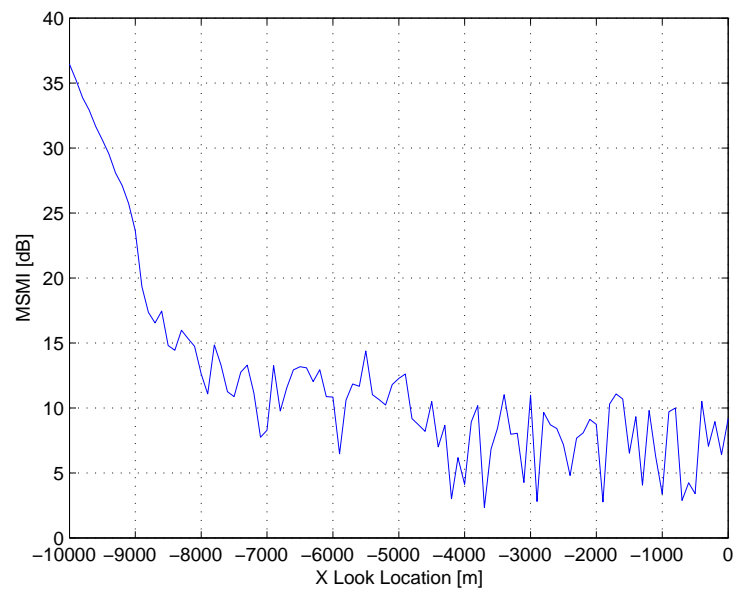

Fig. 5. Optimal processing along the $\mathrm{x}$ direction with a target present at each look point

focusing of the array, horizontal resolution is dominated by the area of the overlap of pulses from each element about the look point. Figure 5 shows the optimal processing of the clutter ball scenario along the $x$ direction with the target moved to each look point. Target resolution is achieved when it is separated from the clutter ball by approximately $5.5 \mathrm{~km}$.

\section{Future WORK}

The data model developed and examples presented here serve to illustrate the potential, but also the preliminary nature of available work in interference cancellation for waveform diverse, distributed apertures. Distributed apertures have the potential to provide extremely fine spatial resolution. However, this advantage comes with the attendant problems of extremely high numbers of grating lobes and/or high sidelobes.

The long term goal of this effort is to develop effective, adaptive interference suppression schemes for waveform diverse distributed apertures. In approaching a similar goal for airborne radar, researchers in the early 1990s developed an effective data model to develop and test signal processing schemes [1]. The work presented in [8] and summarized here presents a similar model for distributed apertures. The model is tested here on waveform diversity achieved via frequency diversity. The key difference between the model in [1] and the one developed here for distributed apertures is that the look points of interest are within the near-field of the array. We use true time delay to focus energy on a chosen point in space.

In this paper, using the data model developed in Section II, we have shown that randomized spacing coupled with frequency diversity are an effective means to suppress grating lobes and high sidelobes. This data model is also used to illustrate the ability to suppress interference and the extremely fine spatial resolution possible.

There are also serious outstanding questions. As shown in [8], one fundamental assumption in using frequency diversity is the need to coherently process across widely spaced frequencies. One approach to deal with this issue is to use schemes recently proposed for cellular communications, such as orthogonal frequency division multiplexing (OFDM) and its multiple access extension, OFDMA. However, this is only possible if the apertures are all within control of the system designer. Recently researchers have developed types of waveforms that optimized for different criteria. Examples are waveforms based on Golay sequences [9] and waveform design, selection and configuration for tracking [10]. The use of such waveforms for multiple applications, including interference suppression and the design of special waveforms optimized for interference suppression are both completely important open research problems. The data model presented here provides a framework to begin this research.

\section{REFERENCES}

[1] J. Ward, "Space-time adaptive processing for airborne radar," MIT Linclon Laboratory, Tech. Rep. F19628-95-C-0002, December 1994.

[2] M. C. Wicks, "Radar the next generation - sensors as robots," in Proc. of 2003 IEEE Internatonal Radar Conference, Sept. 2003, Adelaide, Australia.

[3] H. D. Griffiths and C. J. Baker, "Passive coherent location radar systems. Part 1: performance prediction," IEE Proceedings on Radar, Sonar and Navigation, vol. 152, no. 3, pp. 153-159, June 2005.

[4] R. S. Adve, R. A. Schneible, and R. McMillan, "Adaptive space/frequency processing for distributed apertures," in Proc. of 2003 IEEE Radar Conference, May 2003, Huntsville, AL.

[5] C. Balanis, Antenna Theory: Analysis and Design. John Wiley, 1997.

[6] B. Himed, J. H. Michels, and Y. Zhang, "Bistatic STAP performance analysis in radar applications," in Proc. of the 2001 IEEE Radar Conference, 2001, Atlanta, GA.

[7] P. K. Sanyal, R. D. Brown, M. O. Little, R. A. Schneible, and M. C. Wicks, "Space-time adaptive processing bistatic airborne radar," in Proc. of the 1999 IEEE Radar Conference, 1999, Waltham, MA.

[8] L. Applebaum and R. S. Adve, "Adaptive processing with frequency diverse distributed apertures," in Proc. of the 2nd International Waveform Diversity and Design Conf., Jan. 2006, Kauai, HI.

[9] S. D. Howard, A. R. Calderbank, and W. Moran, "Finite heisenbergweyl groups and golay complementary sequences," in Proc. of the 2nd International Waveform Diversity and Design Conf., Jan. 2006, Kauai, HI.

[10] S. Sira, A. Papandreou-Suppappola, and D. Morrell, "Time-varying waveform selection and configuration for agile sensors in tracking applications," in Proc. of the 2005 International Conf. on Acoustics, Speech and Signal Processing, Mar. 2005, Philadelphia, PA. 